\title{
State-dependent and odor-mediated anemotactic responses of a micro-arthropod on a novel type of locomotion compensator
}

\author{
MERIJN VAN TILBORG \\ University of Amsterdam, Amsterdam, The Netherlands \\ JAN N. C. VAN DER PERS \\ Syntech, Hilversum, The Netherlands \\ and \\ PETER ROESSINGH and MAURICE W. SABELIS \\ University of Amsterdam, Amsterdam, The Netherlands
}

\begin{abstract}
A novel type of locomotion compensator was designed and tested for its use in orientation behavior experiments with a predatory mite. In this apparatus, displacements of the test animal in the twodimensional plane are recorded using video equipment and a servosphere that keeps the animal in focus. The $x$ and $y$ displacements are registeredusing two rotation encoders and are compensated using a pair of servo-motors, in such a way that the animal is always positioned on top of the sphere, yet moves freely. Well-fed and starved predators were tested for their responses to (1) still air, (2) a stimulus-free air flow, (3) an air flow with odors from uninfested Lima bean leaves, and (4) an air flow with odors from Lima bean leaves infested by plant-feeding mites, the prey of the predatory mites. Anemotactic responses of adult Phytoseiulus persimilis females were feeding state dependent. Well-fed predators moved downwind under Treatments 1-3 but moved neither up- nor downwind in the presence of odors from infested plants (Treatment 4). Starved predators moved upwind under all treatments. These results are in agreement with those of earlier studies in a wind tunnel, and therefore, the new type of locomotion compensator (LC-100) offers an excellent method for studying the orientation behavior of micro-arthropods.
\end{abstract}

Animals make use of cues to reach or avoid a target. The cues may contain genuine directional information, or they may modulate the animal's motivation to orient by such a directional cue. There is a wide variety of experimental setups that do not allow a clear discrimination between the directional and the modulated components of orientation behavior. For example, Y-tube olfactometers are designed to demonstrate a relative preference for one stimulus over the other, but there may well be multiple orientation mechanisms that help direct the animal to the stimulus source. This point was made by Kennedy (1977) in relation not only to Y-tubes, but also to a wide variety of poorly analytic designs, such as wind tunnels with nonuniform wind and/or

\footnotetext{
We are thankful to T. D. Wyatt and A. Janssen for reviewing the manuscript, J. Siebring for writing the program used for analyzing the SphereTrack-generated output files, and S. Ragusa for collecting the P. persimilis mites. The co-author J.v.d.P. from Syntech is the developer of the LC-100, used in our experiments. More information about the device can be obtained from http://www.syntech.nl. Correspondence concerning this article should be addressed to M. van Tilborg, Institute for Biodiversity and Ecosystem Dynamics, University of Amsterdam, Kruislaan 320, 1098 SM Amsterdam, The Netherlands (e-mail: tilborg@ science.uva.nl).
}

odor fields. Another drawback shared by these experimental devices is the presence of borders that may affect or even guide the behavior (Sabelis \& Dicke, 1985). Such problems can be avoided by creating arenas that are large relative to the scale of animal movement; however, especially in micro-arthropods, this gives rise to problems in tracking the individuals.

For ambulatory movement in arthropods, a locomotion compensator (servosphere), first described by Kramer $(1975,1976)$, overcomes all of the above problems and allows analytic study of orientation mechanisms. A locomotion compensator is a movement feedback system, with which displacements of the test animal in $x$ and $y$ directions are continuously recorded and compensated, using a pair of servo-motors in such a way that the test animal always walks freely on top of the sphere. Apart from removing the edge effect, the locomotion compensator also has the advantage of fixing the position (but not the movement!) of the test animal, thus enabling careful and controlled manipulations of its direct environment. Locomotion compensators have been used extensively in studies on arthropod orientation behavior (Bell \& Kramer, 1979; Kramer, 1975, 1976; McMahon \& Guerin, 2000; Thiery \& Visser, 1986). The only limitation of the currently avail- 
able locomotion compensators is the need to mount small reflector beads on the back of the test animal for adequate observation of the animal by camera and software. This, however, is a serious problem in studying micro-arthropods. For the predatory mite Phytoseiulus persimilis AthiasHenriot (with a length of $0.5 \mathrm{~mm}$ ), the smallest reflector required for tracking is in the range of its own body size. Clearly, this may have side effects on its natural behavior, and up till now, this limitation has prevented the use of locomotion compensators for well-controlled orientation behavior experiments with micro-arthropods.

In this article, we present a newly designed locomotion compensator, the LC-100 prototype (Syntech, Hilversum, The Netherlands), that is able to track small arthropods without the need to mount a reflector. To test this prototype, we used $P$. persimilis, an acarine predator of the phytophagous spider mite Tetranychus urticae Koch. In reaction to herbivory by spider mites, Lima bean plants produce a specific blend of volatiles, which elicit a behavioral response in the predatory mites (Dicke, Sabelis, Takabayashi, Bruin, \& Posthumus, 1990). Responses of P. persimilis to herbivore-induced plant volatiles have been studied extensively during the last 2 decades in different experimental setups, including Y-tube olfactometers (Dicke et al., 1990; Sabelis \& Van der Baan, 1983) and greenhouse experiments in which the predators were released in the middle of a hexagon of alternately infested and clean plants (Janssen, 1999). In addition to these experiments demonstrating a response to herbivore-induced plant volatiles, there are also a series of published experiments that analyze the orientation response to steep odor gradients in vertical airflow olfactometers (Sabelis, Vermaat, \& Groeneveld, 1984) and to wind direction on a grid (Sabelis \& Van der Weel, 1993) and in a wind tunnel (Van Tilborg, Sabelis, \& Roessingh, 2003). Sabelis and Van der Weel and Van Tilborg et al. found that starved mites always oriented upwind when a stimulus-free air flow or an air flow with odors either from uninfested or spider mite infested Lima bean plants was offered. When well fed, the mites showed negative anemotaxis toward a stimulus-free air flow and an air flow with odors from uninfested Lima bean plants. However, in an air flow with odors from spider mite infested Lima bean plants, the negative anemotaxis disappeared and switched to positive anemotaxis in the experiments of Sabelis and Van der Weel, but to a random walk in those of Van Tilborg et al.

To test the LC-100 prototype of the locomotion compensator, we conducted another set of experiments to analyze anemotaxis in predatory mites, yet with the same treatments as those explored by Sabelis and Van der Weel (1993) and Van Tilborg et al. (2003). Using well-fed and starved predatory mites, four treatments were applied: (1) still air, (2) an air flow free of plant odors, (3) an air flow with odors from uninfested Lima bean leaves, and (4) an air flow with odors from Lima bean leaves infested with two-spotted spider mites. These experiments serve to test whether the orientation response to the wind direction (directional cue) is modulated by the predator's state of starvation (satiation) and/or is mediated by the presence of plant odors (whether herbivore induced or not).

\section{METHOD}

\section{Locomotion Compensator}

The locomotion compensator (LC-100 prototype; Syntech, Hilversum, The Netherlands) used in the present study is essentially a smallscale version of the instrument first described in Kramer (1976), in which a $400-\mathrm{mm}$ sphere was used. The present device consists of a 100-mm-diameter polyphenolic sphere (Saluc S.A., Callenelle, Belgium) placed under a stereo zoom microscope (Olympus SZ4045 TR-CTV) provided with a video tube. At the underside, the sphere is supported by a ball bearing, and it can be rotated in the $x$ and $y$ directions by two servo-motors (Maxon Motor A.G. Sachseln, Switzerland), contacting the sphere at right angles at the equator. On the opposite sides of the motors, two digital incremental rotation encoders (Hengstler GmbH, Aldingen, Germany) are mounted.

The animal under study is placed on top of the sphere and is viewed with a CMOS video camera mounted on the video tube of the microscope. The sphere with the motors, the encoders, and the microscope with the CMOS camera are assembled together in one module. Another module contains a 9-in. TFT video display and the necessary electronics to control the motion of the sphere. The image of the animal is monitored on the TFT screen, and a dedicated image processor converts the $x$ and $y$ displacements of the animal into two analog signals proportional to the displacements at a rate of 25 times per second. We used an image processor that measures the contrast between the animal and the uniform surface of the sphere. Therefore, application of a reflector on the back of the animal, which is necessary in conventional locomotion compensators, is not needed. After amplification and filtering, the two signals control the speed and the direction of the servo-motors in such a way that the test animal is always kept at the center of the image — that is, it always stays on top of the sphere. The tracking behavior of the controller can be adjusted to fit the locomotion pattern of the test animal by adjusting the gain and the feedback of the system.

The signals from the two rotation encoders representing the displacements of the animal in $x$ and $y$ directions are transferred as serial data to a computer at a rate of 10 per second and with a spatial resolution of $0.1 \mathrm{~mm}$. Dedicated software (SphereTrack V.2.0, Syntech, Hilversum, The Netherlands) stores the data as commaseparated text files and reconstructs a real-time track of the displacements of the animal. Two additional digital inputs that can serve as event markers are available. Their binary value is also transmitted at a rate of $10 \mathrm{~Hz}$.

The instrument can be used for tracking very small animals, because a stereo microscope is used to produce the image, allowing large magnifications. The device can be operated with visible and infrared illumination. As a light source, we used eight white-light light-emitting diodes symmetrically positioned in a PVC ring (inner diameter of $190 \mathrm{~mm}$ ). For a schematic representation of the LC-100 prototype, see Figure 1.

Mites

The predatory mites, $P$. persimilis, originated from a wild population in Palermo, Italy, sampled in April 2001, and were reared on detached Lima bean leaves infested with two-spotted spider mite (T. urticae) colonies on water-soaked clay pots in a climate room at $25^{\circ} \mathrm{C}$ and $60 \% \mathrm{RH}$ in our laboratory. The two-spotted spider mites, T. urticae, were reared on Lima bean plants at $24^{\circ} \mathrm{C}$ and $60 \% \mathrm{RH}$ in a climate room and have been in use in the laboratory for more than 10 years.

\section{Experimental Treatments}

In our experiments, we used adult females, all in the oviposition phase and 4-8 days old since the last molt, to ensure a standardized 


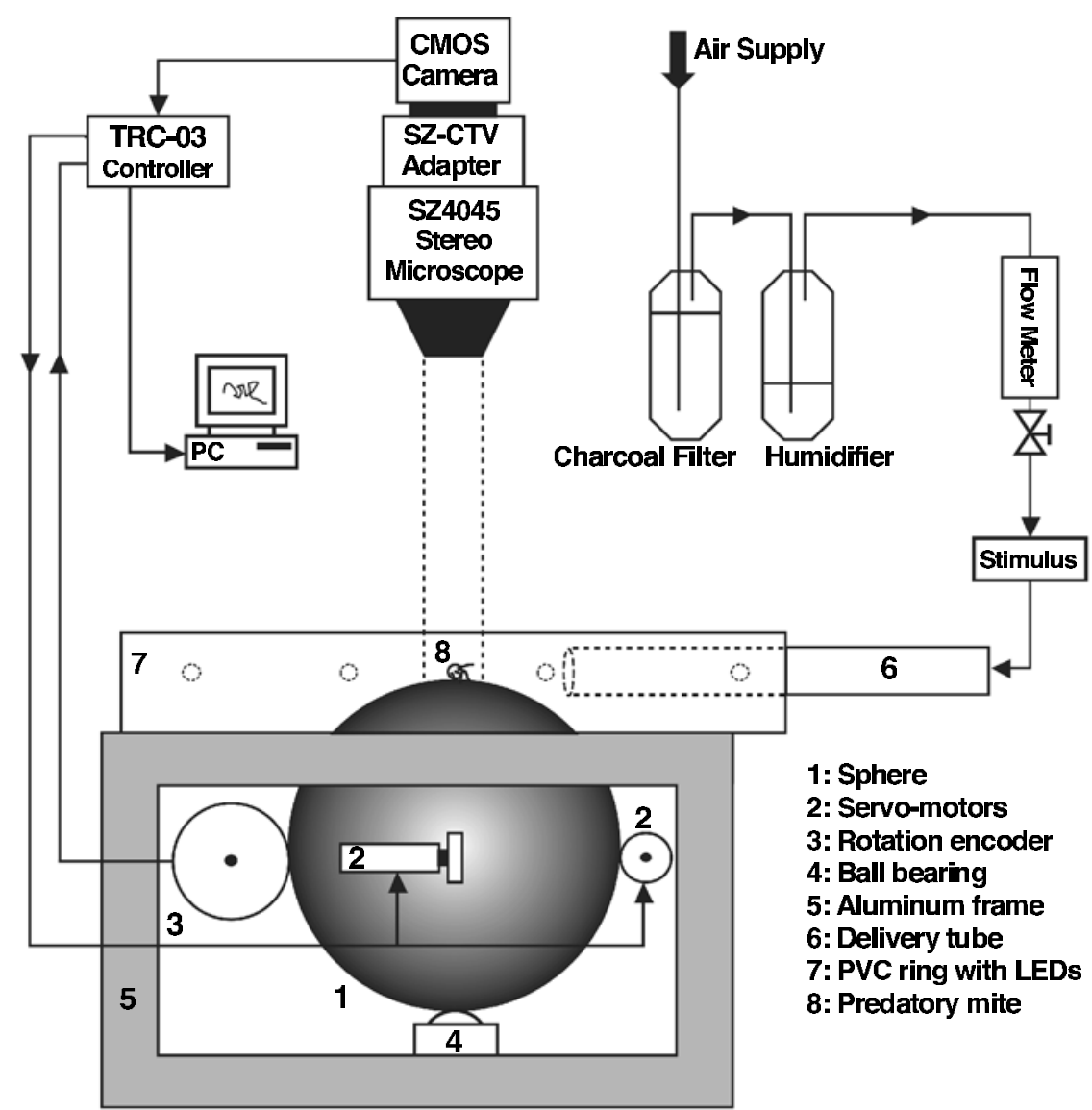

Figure 1. Experimental setup of the locomotion compensator (LC-100 prototy pe) and the odor delivery system.

population of test animals. To obtain well-fed mites for our experiments, a spider mite infested bean leaf with predatory mites was transferred from the culture unit to a Petri dish (provided with a piece of moisturized cotton wool to increase humidity). To obtain starved mites, individual females were transferred from the culture unit into small plastic Eppendorf tubes and were kept without food for 20 $24 \mathrm{~h}$ at $25^{\circ} \mathrm{C}$ and $60 \% \mathrm{RH}$.

Every day of the experiment, either starved or well-fed predatory mites were individually exposed to one of the following four treatments: (1) a stimulus-free still air, (2) a stimulus-free air flow, (3) an air flow with odors from uninfested bean leaves, or (4) an air flow with odors from Lima bean leaves infested with spider mites. For each replicate, a new mite was transferred with a fine camel brush to the top of the sphere. We allowed the mite to acclimatize for $1 \mathrm{~min}$ in still air before the actual experiment started. The walking path of each individual mite was recorded for $5 \mathrm{~min}$, after which the mite was removed. When the LC-100 did not successfully track a mite for $5 \mathrm{~min}$ or the mite did not move at least $5 \mathrm{~cm}$, that replicate was excluded from the data set. After testing 4 mites (each with one treatment), the sphere was cleaned with water and soap and was rinsed with $70 \%$ ethyl alcohol. Thereafter, the same procedure was repeated until a total of 10 replicates per treatment was obtained per day for the starved mites as well as for the well-fed mites. To obtain a total of 30 replicates per feeding state and stimulus condition, the experiments were carried out over a period of 6 days.

\section{Airflow and Stimuli}

Compressed air was passed through a charcoal filter and a flask filled with distilled water to obtain a moisturized clean air flow. The air flow was passed through a flow meter with a needle valve that served to adjust the flow to $0.15-0.25 \mathrm{~m} / \mathrm{sec}$, using flow speed measurements with a hot-wire anemometer at $3 \mathrm{~mm}$ above the walking surface as a reference. Then the flow was passed through a glass cylinder filled either with three to five Lima bean leaves infested with 200-250 adult female spider mites and numerous juveniles and eggs or with three to five uninfested Lima bean leaves. Finally, the air flow ended up in the delivery tube (inner diameter of $15 \mathrm{~mm}$ ), positioned with its mid-axis at the same height as, but approximately $3 \mathrm{~cm}$ away from, the top of the sphere. In the stimulus-free trials, the glass cylinder was removed from the setup. The air flow was laminar, according checks using white smoke $\left(\mathrm{NH}_{4} \mathrm{Cl}\right)$ generated from solutions of household ammonia and $\mathrm{HCl}$ (both $\sim 10 \%$ ). These checks were carried out once per day prior to the experiments, and the sphere and the delivery tube were washed directly after these checks to remove any residues.

\section{Data Analysis}

The output files, generated by SphereTrack V.2.0 (Syntech, Hilversum, The Netherlands) were analyzed by a second program, written in $\mathrm{C}++($ Visual $\mathrm{C}++$ Version 6 and Microsoft Foundation Class Library, Microsoft, Seattle). This imports all individual output files within a directory, calculates the individual vector length and direction of each walking path, and generates a single output file with these parameters. This output file was analyzed using Oriana software for circular statistics (Oriana, 1999).

For each group of test animals with the same feeding state and stimulus condition, the mean vector was calculated, yielding two properties: its direction (mean angle, $\phi$ ) and its length $(r)$. A Raleigh's uni- 
formity test was performed to calculate the probability that the data were distributed in a uniform manner under the null hypothesis. Critical values less than the level of statistical significance $(\alpha=.05$, after Bonferroni correction: $\alpha^{\prime}=n / k ; \alpha^{\prime}=.006$ ) indicate that the data are not distributed uniformly and that there is evidence for preferred direction(s) (Batschelet, 1981).

\section{RESULTS}

In still air, both well-fed and starved predatory mites walked in random directions (Figure 2A, well-fed/still-air, $p>.75, r=.10$; Figure 2E, starved/still-air, $p>.57, r=$ .14). Well-fed mites responded by means of negative anemotaxis to a stimulus-free air flow (Figure 2B, wellfed/stimulus-free, $p<.001, r=.67, \phi=175^{\circ}$ ) and to an air flow with odors from uninfested Lima bean leaves (Figure 2C, well-fed/uninfested bean leaves, $p<.001, r=$ $\left..62, \phi=188^{\circ}\right)$. Well-fed mites lost their anemotactic response and oriented randomly when an air flow with odors from Lima bean leaves infested by spider mites was offered (Figure 2D, well-fed/infested bean leaves, $p>.25$, $r=.22)$. Starved mites responded to an air flow by means of positive anemotaxis, whether odors were present or not (Figure 2F, starved/stimulus-free, $p<.001, r=.50, \phi=$ $326^{\circ}$; Figure $2 \mathrm{G}$, starved/uninfested bean leaves, $p<.001$, $r=.53, \phi=11^{\circ} ;$ Figure $2 \mathrm{H}$, starved/infested bean leaves, $p<.001, r=.61, \phi=11^{\circ}$ ).

\section{DISCUSSION}

The LC-100 prototype proved to be a valuable tool for studying the orientation behavior of small arthropods. Starved mites respond to an air flow by means of positive anemotaxis whether odors are present or not, which is in agreement with the results of Sabelis and Van der Weel (1993) and Van Tilborg et al. (2003). When starved mites perceive stimuli from an upwind source, walking in that direction will bring them closer to the source - that is, into the prey patch. Why starved mites show positive anemotaxis, rather than walking downwind or cross-wind, in the absence of herbivore-induced plant volatiles is not immediately obvious. Sabelis and Schippers (1984) showed theoretically that to find an odor plume under sufficiently variable wind directions, upwind or downwind searching are equivalent but are better strategies than cross-wind searching. Van Tilborg et al. argued that there is a selective advantage for upwind (instead of downwind) searching by starved mites in the absence of odor. This is because it does not require a switch in anemotactic behavior upon entering an odor plume.

Well-fed adult $P$. persimilis females responded to a stimulus-free air flow and to an air flow with odors from uninfested Lima bean leaves by means of negative anemotaxis, which is in agreement with Sabelis and Van der Weel (1993) and Van Tilborg et al. (2003). This behavior is thought to be advantageous to a well-fed predatory mite; being well fed, it must be close to a prey patch (a group of spider mite infested leaves on one or more nearby plants); when it happens to move out of its prey patch at the windward side, it stops perceiving the odor plume, so a negative anemotaxis would bring the mite back to the spider mite patch (Sabelis \& Van der Weel, 1993). Wellfed predatory mites did not show an anemotactic response and oriented in random directions when offered an air flow with odors from Lima bean leaves infested with twospotted spider mites. This is in agreement with the results from Van Tilborg et al. under the same conditions in a wind
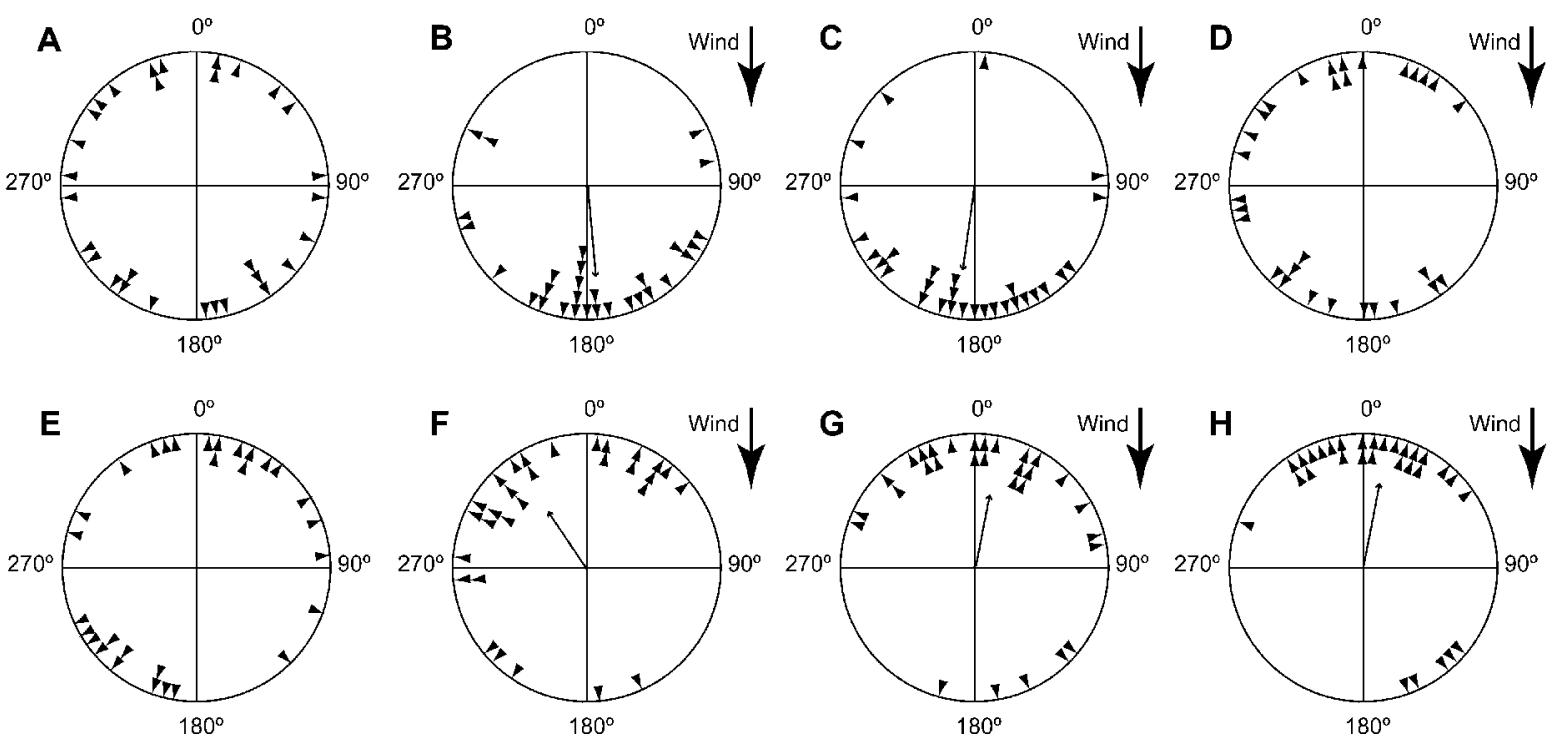

Figure 2. Circular representation of the distribution of the mean direction of walks of well-fed (A, B, C, and D) and starved (E, F, G, and $\mathrm{H}) P$. persimilis mites $(n=30)$ on the locomotion compensator during a 5-min test period. Starved mites were kept for 20-24 $\mathrm{h}$ without food. Stimulus conditions for $A$ and $E$, still air; $B$ and F, stimulus-free air flow; $C$ and $G$, air flow with uninfested Lima bean leaves; and $\mathrm{D}$ and $\mathrm{H}$, air flow with odors from Lima bean leaves infested with two-spotted spider mites. 
tunnel with a laminar air flow. However, it is in contrast with the results from Sabelis and Van der Weel, who, under those conditions, but in another experimental setup (i.e., a grid in an air stream), found a positive anemotactic response of well-fed predatory mites at sufficiently high odor concentrations. Increased odor concentrations did not elicit a positive anemotactic response in our setup (Van Tilborg, Roessingh, \& Sabelis, unpublished). Our setup differed in providing a constant laminar air flow, instead of the turbulent air around the grid in the experiments of Sabelis and Van der Weel. This constant flow may simulate the situation in which the mite is "captured" in the center of an infinite odor field. It may, therefore, lack information from weak gradients in odor concentration and direction, as it may have in the experiments of Sabelis and Van der Weel.

In our tests with the predatory mite $P$. persimilis, we came across two problems in using the LC-100 prototype. The first is the buildup of static electrical charge over time on the sphere that can hinder tiny arthropods such as $P$. persimilis. When this happens, the legs of the mite stick to the sphere, thereby preventing locomotion. To reduce the influence of static electrical charge, we increased humidity by placing trays with water-soaked cotton wool on either side of the sphere. Moreover, we rinsed the sphere after every four replicates. A second problem is caused by airborne dust particles that rain down on the sphere. The relatively larger dust particles may show more contrast to the background (the sphere) than does the test animal. Whenever this happens, the mite will no longer be tracked, and the camera will stay focused on the dust particle instead. This can easily be solved by using a very clean, uncharged sphere and by focusing on the upper body parts of the test animal, so that most of the dust particles stay out of focus. Using these precautions, fewer than $5 \%$ of the trials were interrupted by one of these problems. When larger test animals are used on the sphere, these precautions may be of even less importance.

To our knowledge, the only other locomotion compensator that works without the need of a reflector has been developed by Sakuma (2002). This apparatus is not commercially available and has a larger sphere (505-mm diameter) than the LC-100 (100 mm). The LC-100 prototype proved to be a valuable tool for developing well-controlled experiments on orientation behavior in mites and we expect that it will also prove to be such with other small arthropods.

\section{REFERENCES}

Batschelet, E. (1981). Circular statistics in biology (Mathematics in biology). New York: Academic Press.

Bell, W. J., \& Kramer, E. (1979). Search and anemotactic orientation of cockroaches. Journal of Insect Physiology, 25, 631-640.

Dicke, M., Sabelis, M. W., Takabayashi, J., Bruin, J., \& Posthumus, M. A. (1990). Plant strategies of manipulating predator-prey interactions through allelochemicals: Prospects for application in pest control. Journal of Chemical Ecology, 16, 3091-3118.

JANSSEN, A. (1999). Plants with spider-mite prey attract more predatory mites than clean plants under greenhouse conditions. Entomologia Experimentalis et Applicata, 90, 191-198.

KenNedy, J. S. (1977). Olfactory responses to distant plants and odor sources. In H. H. Shorey \& J. J. McKelvey, Jr. (Eds.), Chemical control of insect behavior: Theory and application (pp. 67-91). New York: Wiley-Interscience.

Kramer, E. (1975). Orientation of the male silkmoth to the sex attractant bombykol. In D. A. Denton \& J. P. Coghlan (Eds.), Olfaction and taste $V$ (pp. 329-355). New York: Academic Press.

KrAMER, E. (1976). The orientation of walking honeybees in odour fields with small concentration gradients. Physiological Entomology, 1, 27-37.

McMahon, C., \& Guerin, P. M. (2000). Response of the tropical bont tick, Amblyomma variegates (Fabricius), to its aggregationattachment pheromone presented in an air stream on a servosphere. Journal of Comparative Physiology A, 186, 95-103.

OrIANA (1999). Oriana v. 1.06 software for circular analysis. Available at http://www.kovcomp/oriana/.

SABELIS, M. W., \& Dicke, M. (1985). Long-range dispersal and searching behavior. In W. Helle \& M. W. Sabelis (Eds.), Spider mites: Their biology, natural enemies and control (Vol. 1B, pp. 141-160). Amsterdam: Elsevier.

Sabelis, M. W., \& SChIPPERS, P. (1984). Variable wind directions and anemotactic strategies of searching for an odour plume. Oecologia, 63, 225-228.

Sabelis, M. W., \& Van der BaAn, H. E. (1983). Location of distant spider mite colonies by phytoseiid predators: Demonstration of specific kairomones emitted by Tetranychus urticae and Panonychus ulmi. Entomologia Experimentalis et Applicata, 33, 303-314.

SABELIS, M. W., \& VAN Der WeEL, J. J. (1993). Anemotactic responses of the predatory mite, Phytoseiulus persimilis Athias-Henriot, and their role in prey finding. Experimental \& Applied Acarology, 17, 521-529.

Sabelis, M. W., Vermaat, J. E., \& Groeneveld,A. (1984). Arrestment responses of the predatory mite, Phytoseiulus persimilis, to steep odour gradients of a kairomone. Physiological Entomology, 9, 437-446.

SAKuma, M. (2002). Virtual reality experiments on a digital servosphere: Guiding male silkworm moths to a virtual odour source. Computers \& Electronics in Agriculture, 35, 243-254.

ThIERY, D., \& VISSER, J. H. (1986). Masking host plant odour in the olfactory orientation of the Colorado potato beetle. Entomologia Experimentalis et Applicata, 41, 165-172.

Van Tilborg, M., Sabelis, M. W., \& Roessingh, P. (2003). Statedependent and odour-mediated anemotactic responses of the predatory mite Phytoseiulus persimilis Athias-Henriot in a wind tunnel. Manuscript submitted for publication. 